\title{
Perfiles de escuelas rurales exitosas: Una propuesta de la psicología educacional ante el cierre de escuelas*
}

\author{
Profiles of successful rural schools: A proposed \\ educational psychology to school closures
}

Recibido: 30 de octubre de 2014| Aceptado: 28 de septiembre de 2015

\author{
DANIELA VERA-BACHMANN ** \\ Universidad Austral de Chile, Chile \\ SONIA SALVO*** \\ Universidad de La Frontera, Chile
}

doi: 10.11144/Javeriana.upsy15-2.pere

Para citar este artículo: Vera-Bachman, D., \& Salvo, S. (2016). Perfiles de escuelas rurales exitosas: Una propuesta de la psicología educacional ante el cierre de escuelas. Universitas Psychologica, 15(2), 25-38. http://dx.doi.org/10.11144/Javeriana.upsy15-2.pere

* Este artículo reporta los resultados obtenidos en la primera etapa de una investigación original que ha sido financiada por CONICYT, Chile. Proyecto FONDECYT N¹1130302, 2013-2015: "Escuelas rurales académicamente exitosas: experiencias susceptibles de replicar para la mejora de la educación rural chilena".

** Doctora en Ciencias de la Educación. Académica Escuela de Psicología. Los Pinos s/n Balneario Pelluco, Puerto Montt, Chile. Teléfono: +56-0652277153. Correo electrónico: danielavera@spm. uach.cl

**** Doctora en Estadística. Universidad de La Frontera, Académica Departamento de Matemática y Estadística. Avenida Francisco Salazar 01145, Temuco, Chile. Teléfono: +56-045-2325678. Correo electrónico: soniasalvo@ufrontera.cl

\section{RES U MEN}

La política pública chilena ha optado por materializar el cierre de escuelas rurales argumentando su baja calidad educativa. Esto ha traído una serie de consecuencias para la comunidad en la que se inserta la escuela. Una forma de revertir esta situación, es mejorando la calidad educativa, para lo cual se identificaron y caracterizaron perfiles de escuelas rurales de alto rendimiento académico según sus resultados en la prueba SIMCE 2011. Se utilizó un análisis multivariado, que incluyó análisis por componentes principales y el método de clasification and regression trees. Los resultados evidenciaron dos perfiles de escuelas rurales exitosas, cuyas principales características se relacionan con la disciplina y la percepción de autoeficacia del estudiante. Estos factores se presentan como importantes elementos a potenciar en los planes de mejoramiento educativo, como alternativa al cierre de escuelas. Palabras clave

educación rural; cierre de escuelas; calidad de la educación; accountability; perfiles de escuelas

\section{A B S T R A C T}

The Chilean public policy has chosen to materialize the closure of rural schools arguing the low quality of its education. This decision has several consequences for the community in which the school is inserted. This situation can be address by improving the quality of education itself. For this purpose, in this study the profiles of rural schools with high academic performance were identified and characterized based on the results of the SIMCE 2011. A multivariate analysis was used including principal component analysis and the method of classification and regression trees. The results showed two profiles of successful rural schools, the main characteristics of them are related to the discipline and self-efficacy of the students. These factors are presented as milestones for the improvement programs for the education (promoted by the Ministry of Education throughout the country), as an alternative to the closure of the rural schools.

Keywords

rural education; closing schools; quality of education; accountability; school profiles 


\section{Introducción}

En los últimos años ha habido un gran debate sobre cómo mejorar la calidad de la educación, y una gran parte de este se ha centrado en los resultados educativos. Este debate ha surgido tras observar que cada vez más recursos ingresan al sistema educativo, sin que eso signifique necesariamente conseguir mejores resultados (Mizala \& Romaguera, 2000). Esta dificultad ha cobrado especial importancia para gran parte de los países (Gershberg, González \& Meade, 2012), puesto que la mayoría de las escuelas que muestran bajos resultados de aprendizaje, se caracterizan generalmente, por sus altos índices de vulnerabilidad y su ubicación en lugares de bajos recursos económicos (Gershberg, Meade \& Andersson, 2009; Kim \& Sunderman, 2004).

La experiencia internacional ha demostrado que una de las formas de responder al problema de las escuelas de bajo rendimiento, es la introducción de incentivos a través de mecanismos de accountability a nivel escolar, como una estrategia para mejorar el desempeño de estas escuelas (Anderson, 2005; Carnoy \& Loeb, 2002; Figlio \& Rouse, 2006; Gershberg et al., 2009; 2012; Jacob, 2005; Reback, 2008; West \& Peterson, 2006). Estos mecanismos, fijan metas mínimas de rendimiento en términos de mejora de los aprendizajes, clasificando a las escuelas según su nivel de cumplimiento y luego aplicando sanciones para aquellos establecimientos que no mejoren su calidad educativa (Brady, 2003; Chiang, 2009; Hanushek \& Raymond, 2005; Spreng, 2005; Springer, 2008).

En el caso de Chile, la inserción de este tipo de mecanismos ha sido más bien reciente. La Ley de Subvención Escolar Preferencial (SEP) (Ley №20.248) es un ejemplo de ello (Ministerio de Educación de Chile, 2008). Esta ley introduce, elementos de evaluación de desempeño sobre las escuelas, según sus resultados de aprendizaje y el cumplimiento de compromisos tomados con base en una evaluación inicial de estos resultados (Contreras \& Corvalán, 2010). Cada escuela debe diseñar e implementar un plan de mejoramiento educativo (PME), considerando acciones en las áreas de gestión curricular, liderazgo escolar, convivencia escolar y/o gestión de recursos (Perticara, Román, \& Selman, 2013; Raczynski, Wieinstein, \& Pascual, 2013). Si las metas establecidas no son alcanzadas, la ley contempla una serie de medidas que, eventualmente, pueden derivar en la revocación del reconocimiento oficial de los establecimientos, es decir, su cierre (Elacqua, Santos, Salazar, \& Brunner, 2011).

El cierre de la escuela, es la medida más extrema que se puede aplicar a un establecimiento educacional que persiste en mostrar bajos niveles de aprendizaje. La idea que subyace a esta medida, estima que esta es la sanción más efectiva para establecimientos que demuestran ser crónicamente deficientes y que simplemente no tienen solución (Brady, 2003). Ahora bien, se sostiene que con la clausura de estas escuelas, los estudiantes podrán ser transferidos a establecimientos que realmente tengan mejor desempeño y además, se aplicarán incentivos para que otras escuelas de bajo rendimiento mejoren ante la amenaza de ser cerradas (Elacqua \& Martínez, 2011; Smarick, 2010).

Pero iqué pasa en Chile actualmente? Hasta el momento, ha estado ausente el análisis de impacto del posible cierre de establecimientos considerados deficientes por el sistema educativo en la discusión sobre políticas públicas en educación, (Elacqua et al., 2011), sobre todo para el caso de la educación rural en donde las investigaciones son escasas y se dispone de poca información al respecto (ÁbergBrentsson, 2009; Arnold, Newman, Gaddy, \& Dean, 2005; Hargreaves, 2009; Hargreaves, Kvalsund, \& Galton, 2009; Núñez, Solís, \& Soto, 2014; Sherwood, 2000; Vera, Osses, \& Shiefelbein, 2012). Entonces surgen preguntas como las siguientes: ¿Podrá el sistema educativo garantizar el acceso de los estudiantes a alternativas de calidad, que les permitan obtener mejores resultados de aprendizaje? ¿Qué ocurrirá con los estudiantes de escuelas ubicadas en zonas rurales?

Actualmente, la red escolar cuenta con aproximadamente 3,835 escuelas rurales distribuidas en todo Chile (Araya et al., 2012; Ministerio de Educación de Chile, 2010; Núñez et al., 2014). Se trata de escuelas que, en su mayoría, han sido criticadas por sus resultados educativos constantemente bajo el promedio nacional medido por el Sistema de Medi- 
ción de la Calidad de la Educación (SIMCE, 2011). Además, se suma el cuestionamiento respecto de su escaso equipamiento (Atchoarena \& Gasperini, 2004), su menor tamaño (Araya et al., 2012; Kearns, Lewis, McCreanor \& Witten, 2009) y su reducido número de matrícula producto de la disminución de la población en zonas rurales, fenómeno que se repite en varios países del mundo (Arnold et al., 2005; Donoso \& Arias, 2012; Hargreaves, 2009; Kearns, et al., 2009; Kalaoja \& Pietarinen, 2009; Núñez, et al., 2014; Smit \& Humpert, 2012). Esto último, provocaría un incremento en el gasto por estudiante (Áberg-Brentsson, 2009; Gallego, Rodríguez \& Sauma, 2007; 2010), lo que se traduce en un déficit financiero que afectaría directamente al sistema educativo (Fantuzzi, 2008).

Pese a lo anterior, estudios han demostrado que una fracción importante de las escuelas rurales chilenas no tiene alternativas de calidad cercanas, incluso considerando un radio de $5 \mathrm{Km}$. Lo interesante de estos resultados, es que aun cuando la matrícula es escasa, el gasto por estudiante es alto y los resultados educativos son bajos, existe un número importante de escuelas rurales que no tienen alternativas de calidad cercanas (Elacqua \& Martínez, 2011; Vera, 2013; Vera, Salvo \& Zunino, 2013). Esto indica, que si bien el cierre puede ser una medida aplicable en mercados educacionales con diversidad de opciones, en el caso de aquellas escuelas que no cuentan con mejores alternativas cercanas o donde no existen subsidios de transporte para asistir a escuelas lejanas de mejores resultados, el cierre de escuelas rurales, no parece la opción más adecuada (Elacqua, Martínez, Santos \& Urbina, 2013; Elacqua et al., 2011).

Frente a este escenario parece relevante preguntarse cuáles son los caminos a seguir, sobre todo recordando que la escuela rural, es la institución más extendida territorialmente (Ávila, 2010; Vera, 2013; Vera, Salvo et al., 2013). Su acción alcanza a las zonas de menor grado de desarrollo, donde los procesos de despoblamiento hacen de las escuelas un nodo básico de sus débiles redes institucionales, permitiendo mantener articulados socialmente a esos territorios (Delich \& Iaies, 2009; Hargreaves et al., 2009; Núñez et al., 2014). En los sectores rurales desfavorecidos, esta interacción de la escuela con la comunidad y otras organizaciones del medio, termina resultando un elemento fundamental en la construcción y generación de estas formas cooperativas y asociativas de capital social, que se transforman en recursos y beneficios para sus habitantes (Barley $\&$ Beesley, 2007; Hargreaves, 2009; Kearns et al., 2009; Kalaoja \& Pietarinen, 2009; Kovács, 2012).

Considerando la relevancia de lo mencionado y la eventual posibilidad de cierre de las escuelas rurales debido a lo planteado por la ley SEP, resulta de suma importancia generar evidencia que permita fortalecer los planes de mejoramiento educativo, señalando con claridad cuáles son las áreas prioritarias a intervenir en la educación rural (Vera, 2013). De esta forma, contar con información que permita identificar cuáles son las escuelas rurales de alto rendimiento y qué variables explican sus resultados, permite tomar decisiones basadas en la evidencia para mejorar la calidad educativa de aquellas escuelas con bajos rendimientos, evitando su cierre (Barley \& Beesley, 2007; Valenzuela \& Allende, 2014; Vera, Salvo et al., 2013).

Lo anterior es de importancia debido a que el cierre de la escuela rural, podría significar la pérdida de uno de los pocos espacios de interacción del mundo campesino (Hargreaves et al., 2009; Núñez, et al., 2014). Esto, podría facilitar la deserción escolar, la desarticulación social y los riesgos de desertización social de los territorios rurales, solo por nombrar algunas de las eventuales consecuencias (Hargreaves, 2009; Kearns et al., 2009; Kalaoja \& Pietarinen, 2009; Kovács, 2012).

Por esta razón, la presente investigación tiene como objetivo principal identificar y caracterizar perfiles de escuelas rurales chilenas de alto rendimiento académico. Contar con este tipo de información, resulta fundamental a la hora de evaluar la implementación efectiva de la política educativa.

\section{Método}

Para caracterizar los perfiles de las escuelas rurales de alto rendimiento según sus resultados en la prueba SIMCE aplicada a cuarto año básico en el año 2011, se desarrolló un estudio que abarcó un 
análisis exploratorio y un análisis multivariado. Los análisis de los datos fueron realizados con el lenguaje de programación estadística R (R-Development Core Team, 2011).

\section{SIMCE 2011}

El SIMCE es el Sistema Nacional de Evaluación de resultados de aprendizaje del Ministerio de Educación de Chile. Su propósito principal es contribuir al mejoramiento de la calidad y equidad de la educación, informando sobre el desempeño de los estudiantes en diferentes subsectores del currículum nacional, y relacionándolos con el contexto escolar y social en el que ellos aprenden (SIMCE, 2011).

Actualmente, las pruebas SIMCE evalúan el logro de los Objetivos Fundamentales y Contenidos Mínimos Obligatorios (OF-CMO) del marco curricular vigente en diferentes subsectores de aprendizaje. El SIMCE es una medición a nivel nacional que se aplica anualmente a los estudiantes de un determinado grado en los entornos urbano y rural. En el caso de $4^{\circ}$ Básico son evaluados los subsectores de lenguaje, matemáticas y ciencias naturales (SIMCE, 2011). Además, el SIMCE recoge información sobre profesores, estudiantes y padres y apoderados a través de cuestionarios de contexto. Esta información se utiliza para analizar los resultados (SIMCE, 2011).

Los datos del SIMCE son públicos para investigación y obtenidos previo acuerdo de confidencialidad. En el año 2011 el SIMCE fue aplicado a 18,455 niños de 1,507 escuelas rurales distribuidas en todo el territorio nacional.

En la Tabla 1 se presentan los principales estadísticos de los rendimientos SIMCE de cuarto básico obtenidos por las escuelas rurales en el año 2011. Respecto a los resultados nacionales (SIMCE, 2011), los puntajes obtenidos por las escuelas rurales se encuentran nueve puntos por debajo en lectura, 16 puntos por debajo en matemática y 18 puntos por debajo en ciencias naturales.

\section{Índices}

A partir de los cuestionarios de contexto del Ministerio de Educación de Chile (2011) y las características de la información con que se trabajó, se hizo necesaria, la reducción dimensional de los datos. Por esta razón, se construyeron una serie de índices (Tabla 2) utilizando análisis por componentes principales (Hotelling, 1933). Este análisis tiene por objetivo representar adecuadamente la información con un número menor de variables construidas como combinaciones lineales de las originales. Su utilidad consiste en que permite representar óptimamente observaciones de un espacio general $\mathrm{p}$-dimensional en un espacio de dimensión pequeña, y además permite transformar las variables originales, en nuevas variables, facilitando la interpretación de los datos.

\section{Análisis de datos}

El rendimiento de los estudiantes en los tres subsectores evaluados fue reducido a un solo indicador mediante el promedio por estudiante. Para cada escuela fue obtenido un rendimiento promedio como indicador de su desempeño, el que es utilizado comúnmente para los rankings nacionales (Valenzuela \& Allende, 2014).

TABLA 1

Principales estadísticos de las pruebas SIMCE

\begin{tabular}{cccc}
\hline & Lectura & Matemáticas & Ciencias \\
\hline Media & 258.5 & 243.5 & 241.9 \\
DT & 27.1 & 31.3 & 27.8 \\
Min & 164.9 & 112.2 & 154.1 \\
Max & 357.4 & 366.0 & 325.1
\end{tabular}

Fuente: elaboración propia 
TABLA 2

Descripción y caracterización de los Índices construidos para la investigación

\begin{tabular}{|c|c|c|}
\hline Índice & Preguntas & Descripción \\
\hline Nivel socioeconómico & $\begin{array}{l}\text { PA08Q01, PA09Q01 y } \\
\text { PA10Q01 }\end{array}$ & $\begin{array}{l}\text { Referente a la escolaridad de los padres y } \\
\text { los ingresos familiares }\end{array}$ \\
\hline Capital cultural & $\begin{array}{l}\text { PA08Q01, PA09Q01, PA10Q01, } \\
\text { PA10Q07 }\end{array}$ & $\begin{array}{l}\text { Referente a la escolaridad de los padres, sus } \\
\text { ingresos y la cantidad de libros en el hogar. }\end{array}$ \\
\hline $\begin{array}{l}\text { Percepción de autoeficacia del } \\
\text { estudiante }\end{array}$ & $\begin{array}{l}\text { ST06Q01, ST06Q02, ST06Q05, } \\
\text { ST06Q07 }\end{array}$ & $\begin{array}{l}\text { Referente a la percepción del estudiante, } \\
\text { respecto de ser capaz de aprender de la } \\
\text { misma forma que sus compañeros y de } \\
\text { entender los contenidos, pese a que estos } \\
\text { sean dificiles y requieran de mayor esfuerzo. }\end{array}$ \\
\hline Disciplina al interior del aula & PR13Q02, PR13Q06, PR13Q07 & $\begin{array}{l}\text { Da cuenta de conductas de respeto entre } \\
\text { estudiantes, al desarrollar un trabajo } \\
\text { siguiendo las instrucciones del profesor y } \\
\text { al mantener el orden dentro del aula de } \\
\text { clases. }\end{array}$ \\
\hline $\begin{array}{l}\text { Capacidad del profesor para manejar } \\
\text { la disciplina al interior del aula }\end{array}$ & ST12Q04, ST12Q05, ST12Q06 & $\begin{array}{l}\text { Referente a la percepción del estudiante } \\
\text { respecto de la capacidad del profesor para } \\
\text { manejar conductas disrruptivas al interior } \\
\text { de la clase. }\end{array}$ \\
\hline $\begin{array}{l}\text { Seguridad del profesor respecto de su } \\
\text { preparación profesional }\end{array}$ & $\begin{array}{l}\text { PR20Q01, PR20Q02, PR20Q03, } \\
\text { PR20Q04, PR20Q05, PR20Q06 }\end{array}$ & $\begin{array}{l}\text { Referente a cuán preparado se siente el } \\
\text { profesor para que aprendan los estudiantes } \\
\text { con baja motivación, con dificultades de } \\
\text { aprendizaje, con problemas económicos, } \\
\text { con dificultades conductuales y problemas } \\
\text { emocionales. }\end{array}$ \\
\hline $\begin{array}{l}\text { Dificultades conductuales al interior } \\
\text { de la escuela }\end{array}$ & $\begin{array}{l}\text { PR13Q01, PR13Q03, PR13Q04, } \\
\text { PR13Q05 }\end{array}$ & $\begin{array}{l}\text { Referente a la presencia de } \\
\text { comportamientos disrruptivos al interior } \\
\text { de la escuela, tales como peleas durante } \\
\text { las clases, trato irrespetuoso hacia los } \\
\text { profesores, clases que se interrumpen } \\
\text { frecuentemente, etc. }\end{array}$ \\
\hline
\end{tabular}

$\mathrm{ST}=$ Cuestionario de estudiante, $\mathrm{PR}=$ Cuestionario de profesores, $\mathrm{PA}=$ Cuestionario de Padres. El primer número corresponde a la pregunta y el segundo al ítem respondido.

Fuente: elaboración propia

Los perfiles de escuelas rurales fueron obtenidos mediante el análisis multivariado CART (Classification And Regression Trees). Este análisis corresponde a un enfoque exploratorio de extracción de relaciones de dependencia, tipo jerárquica, entre una variable de respuesta y un conjunto de variables influyentes (Breiman, Freidman, Olshen $\&$ Stone, 1984). De este modo, son seleccionadas las variables que explican el rendimiento buscando la mayor separabilidad entre grupos.

La descripción de los perfiles corresponde a las variables seleccionadas para los grupos de alto rendimiento según sus resultados SIMCE 2011. Estos grupos corresponden a aquellos que se encuentran sobre los resultados promedio obtenidos por el grupo de referencia (OECD, 2011), en este caso el resultado SIMCE a nivel nacional.

\section{Resultados}

Índices

En la Tabla 3, se presentan los índices construidos y el porcentaje de varianza explicada para cada uno de ellos. En general, los índices alcanzaron un buen nivel de extracción de variabilidad, superior al 51\%. La mayor extracción fue observada en el índice de disciplina al interior del aula y en el de nivel socioeconómico. 
TABLA 3

Índices construidos para la investigación y su varianza explicada

\begin{tabular}{lc}
\hline \multicolumn{1}{c}{ Índice } & Varianza explicada \\
\hline Nivel socioeconomico & $62 \%$ \\
Capital cultural & $60 \%$ \\
Percepción de autoeficacia del estudiante & $51 \%$ \\
Disciplina al interior del aula & $63 \%$ \\
Capacidad del profesor para manejar la disciplina al interior del aula & $52 \%$ \\
Seguridad del profesor respecto de su preparación profesional & $58 \%$ \\
Dificultades conductuales al interior del aula & $59 \%$ \\
\hline
\end{tabular}

Fuente: elaboración propia

\section{Perfiles de escuelas rurales de alto rendimiento}

Desde el análisis CART fueron obtenidas ocho variables relevantes: disciplina al interior del aula, percepción de autoeficacia del estudiante, capital cultural, nivel socio-económico, subvención escolar total percibida el año 2011, dificultades conductuales al interior de la escuela, seguridad del profesor respecto de su preparación profesional y capacidad del profesor para manejar la disciplina al interior del aula.

En la Tabla 4 se presenta el nivel de explicación de cada una de las variables. La percepción de autoeficacia del estudiante fue la variable más importante (explicando un $42 \%$ de variabilidad), seguido de la disciplina al interior del aula (15\%), el nivel socioeconómico (12\%) y el capital cultural (8.5\%). En conjunto, estas cuatro variables explican el $78.2 \%$ del rendimiento promedio obtenido por las escuelas rurales en la prueba SIMCE 2011.
La segmentación de las variables explicativas permitió la formación de 19 nodos terminales que dieron origen a seis grupos en total, como lo muestra la Figura 1. Los nodos comienzan a formarse desde el nodo raíz, el que se separa según las variables más importantes que caracterizan cada grupo de escuelas. Para este caso, el nodo raíz está conformado por 1,507 escuelas, y fue dividido de acuerdo al índice de autoeficacia del estudiante (el índice que obtiene la mayor separación de escuelas). Por ejemplo, el nodo hijo izquierdo agrupó a 576 escuelas con nivel de autoeficacia de un estudiante que no superaban en el 30\% inferior de las escuelas y anotó 239 puntos de logro. Así, sucesivamente se evidencian las características según cada rama proveniente desde el nodo que le precede.

De los 19 nodos terminales, ocho fueron mayores de $0.5 \mathrm{DT}$ por encima del rendimiento promedio y fueron designadas como escuelas de alto rendimiento (Vera, 2013). Tres de estos nodos se

TABLA 4

Variables relevantes para explicar los resultados SIMCE en los dos grupos de escuelas rurales de alto rendimiento.

\begin{tabular}{lc}
\hline \multicolumn{2}{c}{ Variable } \\
\hline Percepción de autoeficacia del estudiante & $42 \%$ \\
Disciplina al interior del aula & $15 \%$ \\
Nivel socioeconomico & $12 \%$ \\
Capital cultural & $8 \%$ \\
Capacidad del profesor para manejar la disciplina al interior del aula & $5 \%$ \\
Dificultades conductuales al interior del aula & $4 \%$ \\
Subvención escolar total percibida el año 2011 & $3 \%$ \\
Seguridad del profesor respecto de su preparación profesional & $1 \%$ \\
\hline
\end{tabular}

Fuente: elaboración propia 


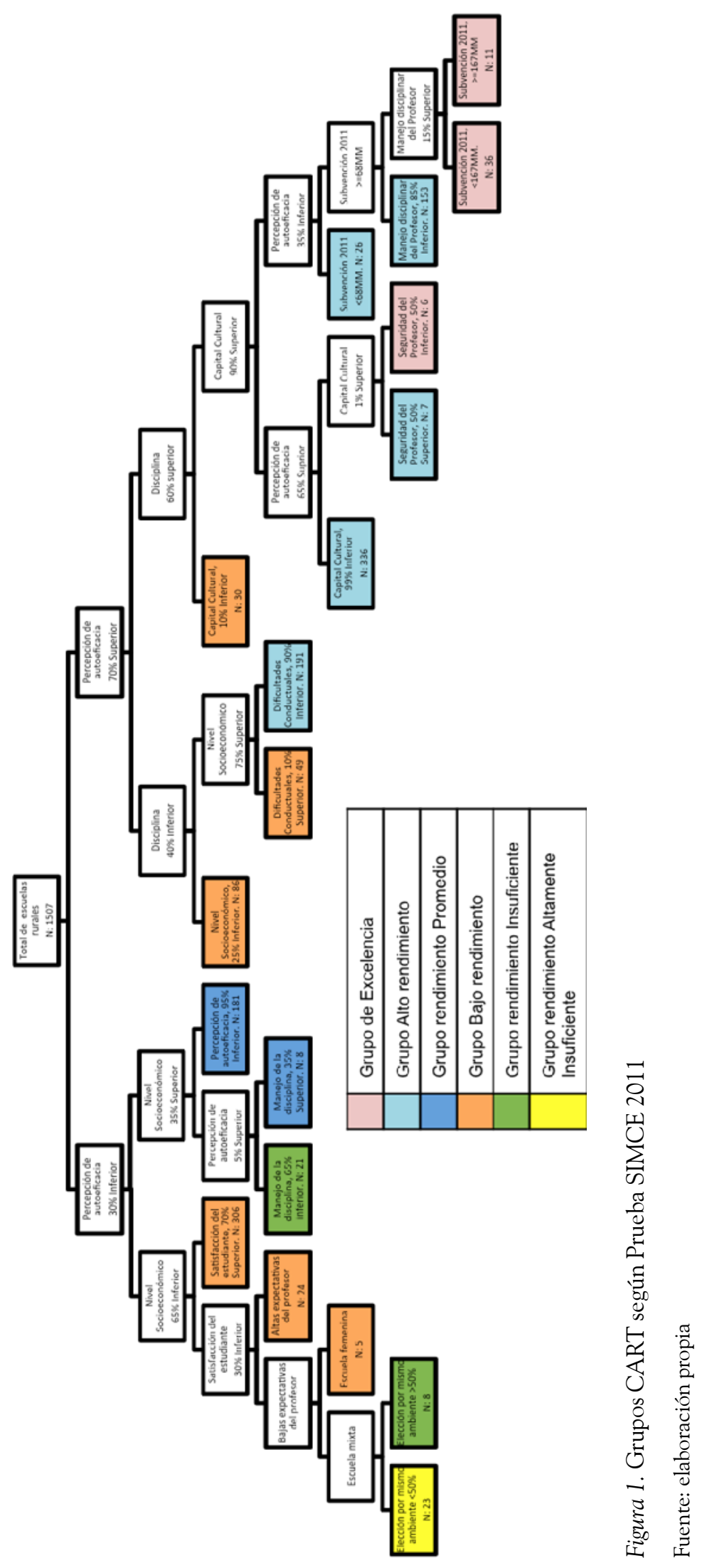


catalogaron como Grupo de Escuelas de Excelencia, compuesto por 53 escuelas, correspondientes al 3.5\% del total de escuelas rurales que rindieron SIMCE 2011, y cuyo desempeño en dicha prueba fue igual o superior a los 283 puntos; y los cinco nodos restantes se catalogaron como Grupo de buen rendimiento, compuesto por 713 escuelas rurales, correspondientes al $47.3 \%$ del total, con un rendimiento SIMCE igual o superior a los 266 puntos.

El perfil de escuelas rurales de excelencia se caracterizó por un alto nivel de percepción de autoeficacia de sus estudiantes (dentro del 70\% de mayor autoeficacia), es decir, estudiantes cuya percepción y valoración de sí mismos, los hace poseedores de la capacidad y recursos personales necesarios para hacer frente a las diversas situaciones de su vida escolar. Además, este perfil presentó una buena disciplina escolar al interior del aula (dentro del 60\% de mejor disciplina), con un alto índice de capital cultural (dentro del $90 \%$ con mayor capital cultural), con una subvención escolar total superior a US $\$ 143,000$ y con profesores altamente capacitados en el manejo de la disciplina al interior del aula (dentro del 15\% de mayor capacidad).

Por su parte, el perfil de escuelas rurales de buen rendimiento se caracterizó por un alto nivel de percepción de autoeficacia del estudiante (en el 70\% de mayor autoeficacia), con buena disciplina al interior del aula (dentro del 60\% de mejor disciplina), lo que significa que son establecimientos en donde existe respeto entre los estudiantes y las clases se realizan en orden y siguiendo las instrucciones del profesor; y con un alto capital cultural (dentro del $90 \%$ con mayor capital cultural), lo que implica presencia de padres y madres con mayores niveles educativos, ingresos más altos y mayor acceso a recursos, que le permiten al estudiante potenciar su proceso de enseñanza-aprendizaje.

\section{Conclusiones}

Si bien la literatura ha planteado que una escuela constituye una ventaja para una comunidad rural (Kearns et al., 2009), en el caso de Chile, los bajos resultados educativos, el menor tamaño de los establecimientos (Araya et al., 2012), su reducido número de matrícula (Kalaoja \& Pietarinen, 2009; Smit \& Humpert, 2012) y su alto gasto por alumno (Áberg-Brentsson, 2009; Gallego et al., 2007; 2010), ha vuelto a la escuela rural vulnerable frente a la implementación de una política de cierre (Núñez et al., 2014).

No obstante lo anterior, existen estudios (Elacqua \& Martínez, 2011; Elacqua et al., 2011) nacionales, que demuestran que un porcentaje importante de escuelas rurales no tiene alternativas cercanas de mejor calidad (Vera, 2013, Vera, Salvo et al., 2013). Por tanto, cerrar una escuela rural en estas condiciones, implicaría aumentar los tiempos de traslado de los alumnos y asumir un nuevo gasto asociado a transporte (Araya et al., 2012). Además, aunque se contase con un bono que permita mitigar estos gastos, la capacidad de absorción de matrícula que presentarían los nuevos establecimientos, seguiría siendo un elemento contextual determinante a la hora de decidir la viabilidad del cierre.

Otro aspecto relevante a considerar es la relación escuela-comunidad. Por un lado, existe evidencia de que la comunidad rural supone un aporte a la educación (Durston, 2002) y por otro, se plantea el rol fundamental de la escuela en el fortalecimiento y desarrollo comunitario (Hargreaves, 2009; Kearns et al., 2009; Kalaoja \& Pietarinen, 2009; Kovács, 2012; Núñez et al., 2014).

En el caso de la educación rural, al generar espacios que permiten integrar la escuela y la comunidad, se propicia la oportunidad de que profesores y padres interactúen más fuertemente. Esta integración permite que los profesores puedan manejar, tanto los códigos culturales como los conocimientos que imperan en la casa de los niños, aprovechando mejor lo que estos ya saben, como base para nuevas enseñanzas. En el ámbito educativo rural, la evidencia muestra que las escuelas son más efectivas cuando los padres y la comunidad participan conjuntamente del proceso educativo (Meneses \& Mominó, 2008).

Además, la investigación indica que la educación promueve el desarrollo de capital social a través de dos aspectos fundamentales; propiciando espacios para que los estudiantes puedan practicar elementos del capital social, tales como la participa- 
ción y la reciprocidad; y ofreciendo instancias para actividades propias de la comunidad al interior de las escuelas (Kearns et al., 2009). De este modo, el beneficio se propicia en una relación virtuosa, en donde la interacción escuela-comunidad, genera aportes tanto educativos como locales o comunitarios (Atchoarena \& Gasperini, 2004; Boix, 2003; Feu, 2003; Riella \& Vitelli, 2005). En consecuencia, el cierre de la escuela rural, significaría la pérdida de uno de los principales espacios de reunión de la comunidad rural (Hargreaves et al., 2009).

En síntesis, si bien el cierre puede ser una medida aplicable en mercados educacionales con diversidad de opciones, en el caso de aquellas escuelas que no cuentan con mejores alternativas cercanas, donde no existen subsidios de transporte a escuelas de mejores resultados, y donde su relación con la comunidad es preponderante, el cierre de escuelas rurales, no parece ser la alternativa más adecuada.

En este sentido, la evidencia encontrada a partir de los resultados de la presente investigación, muestra importantes luces para mejorar la política vigente y encontrar alternativas al cierre de escuelas.

Al contrario de lo que se cree comúnmente, en coherencia con los perfiles de escuelas rurales exitosas, los resultados de este estudio, demostraron que la mejora no siempre está asociada al nivel socioeconómico o a la asignación de mayores recursos. Por el contrario, demuestran que una escuela, puede mejorar el rendimiento de sus estudiantes de forma significativa, si trabaja mejorando curricular y didácticamente la formación integral del estudiante, incluyendo la importancia de variables psicológicas como la percepción de autoeficacia; trabajando la convivencia escolar, especialmente la relevancia de una buena disciplina al interior de la sala de clases; fomentando la competencia del profesor al interior del aula, entre otras.

Los resultados muestran la existencia de grupos de alto rendimiento en escuelas rurales, a partir de los cuales es posible extraer lecciones que podrían ser implementadas en futuros planes de mejora. En estos grupos, fueron ocho las variables que explicaron los resultados SIMCE. Dentro de ellas, las más importantes fueron la percepción de autoeficacia del estudiante, la disciplina al interior del aula, el nivel socioeconómico y el capital cultural. Si bien estas dos últimas variables resultan difíciles de intervenir en el corto plazo para la mejora educativa, el trabajo con las dos primeras - que en su conjunto explican más del $57 \%$ de los resultados obtenidos por las escuelas rurales - podrían reportar resultados factibles de ser evidenciados en el corto plazo, de ahí su importancia.

Lo relevante de estos hallazgos, es que aun cuando el nivel socio-económico sigue apareciendo como una variable explicativa del rendimiento educativo (Anderson, Chui \& Yore, 2010; Willms, 2010; Coll, Dahsah, \& Faikhamta, 2010; Oreiro \& Valenzuela, 2011; Demir \& Depren, 2010; Cordero, Crespo, \& Santín 2010; De Jorge \& Santín, 2010), en escuelas rurales de alto rendimiento, no da cuenta necesariamente de mejores resultados de aprendizaje. De este modo, aún en condiciones de pobreza y aislamiento, el rol del profesor en cuanto a clima escolar y el desarrollo de competencias transversales en el estudiante resultan clave para la mejora educativa (Arshad, Ahmad, Tahira, \& Ahmad, 2011), dos factores profusamente estudiados desde la psicología educacional.

Dado lo anterior, se sugiere fortalecer la política pública desde las orientaciones específicas que entrega el Ministerio de Educación para el diseño de los planes de mejoramiento educativo de escuelas rurales (Vera, 2013), contemplados dentro de los compromisos que establece la Ley SEP. Dado que cada escuela debe diseñar e implementar un plan de mejora, se sugiere focalizar los esfuerzos considerando los siguientes elementos, susceptibles de ser replicados a través de las instancias de intercambio en los microcentros rurales (Vera, Salvo et al., 2013).

En el área de gestión curricular, se sugiere generar líneas orientadas específicamente a fortalecer la percepción de autoeficacia del estudiante, ya sea en el curriculum de forma transversal y/o en talleres especializados para trabajar dicha temática, sobre todo considerando la masiva inclusión de psicólogos al sistema educativo nacional. Algunas investigaciones han demostrado la relevancia de esta variable y su influencia en la motivación, el interés de los estudiantes por sus tareas, la persistencia en los objetivos que se fijan a nivel escolar, las decisiones 
que toman, el uso de habilidades cognitivas, metacognitivas y estrategias de autorregulación, y en el rendimiento académico (Carmichael \& Taylor, 2005; Pajares, 2003; Schunk, 2003). Por tanto, la evidencia demuestra que la autoeficacia juega un rol central en relación con los logros de los estudiantes, la motivación y el aprendizaje (van Dinther, Dochy $\&$ Segers, 2011).

Por su parte, en el área de convivencia escolar, es posible generar líneas orientadas a favorecer un buen clima escolar, con actividades centradas en una buena disciplina al interior del aula y en la capacidad de manejo del profesor respecto de este aspecto, sobre todo en la enseñanza multigrado. Elementos como estos han demostrado tener un fuerte impacto en el rendimiento académico, especialmente, en las escuelas de alta pobreza (Hopson \& Lee, 2011; Moreno, Estévez, Murgui, \& Musitu, 2009; Villalta \& Saavedra, 2012). En este sentido la evidencia sugiere enfatizar en estrategias que promuevan la cooperación y respeto entre los estudiantes, y mejores relaciones estudiante-profesor (Álvarez, Álvarez-García, González-Castro, Núñez \& González-Pienda, 2006; Arón, Milicic, \& Armijo, 2012; Cuthrell, Stapleton, \& Ledford, 2010; García-Hierro \& Cubo, 2009).

Por último, se considera relevante dados los resultados observados - como parte de la relación entre la escuela y su comunidad- promover la nivelación de estudios en aquellos padres y apoderados que aún no han terminado los años de escolaridad obligatoria. De esta forma es posible mejorar el capital cultural de las familias, optar a mejores ingresos y facilitar la movilidad social de las mismas, lo que influye además en la mejora de los resultados académicos de sus hijos (Anderson et al., 2010; Kalender \& Berberoglu, 2009).

$\mathrm{Si}$ bien se reconoce, que algunas de estas variables han sido incorporadas en los actuales lineamientos educativos, aún quedan elementos por perfeccionar para mejorar el rendimiento de las escuelas rurales. No obstante, se debe tener en cuenta para este punto, que en educación y especialmente en educación rural, no existen recetas sino más bien orientaciones, dado que la mayor necesidad sigue siendo subsanar el alto nivel de inequidad social, lo que sin duda cambiaría el actual panorama educativo (Vera, 2013).

En síntesis, respondiendo al objetivo central de esta investigación, es posible señalar que existen importantes razones para buscar alternativas al cierre de escuelas. La existencia de experiencias exitosas en términos educativos y su factibilidad de ser replicadas a nivel nacional, demuestran una importante alternativa para la mejora educativa.

No considerar lo anterior, podría implicar continuar con planes de mejora que incluyen intervenciones que implican altos costos y que no siempre se reflejan en un cambio positivo en los resultados (Vera, 2013). El riesgo que se corre es mantener resultados de aprendizaje insuficientes, arriesgando el cierre de la escuela.

No obstante lo anterior, contar con información adicional que permita seguir mejorando los resultados educativos y la viabilidad de la implementación de las políticas educativas, sigue siendo una necesidad.

Por una parte, se requiere contar con mayor información respecto de la relación escuela-comunidad y los efectos en los procesos y resultados educativos a nivel rural. Lo que existe son estudios de caso, con evidencia no generalizable y que requiere de mayor extensión. La escasa teorización al respecto, hace que en ocasiones, se pase por alto la importancia del "paisaje" social en la educación (Corvalán, 2004).

Por otro lado, se hace necesario contar con información respecto de cómo influye la ruralidad en aspectos relativos a la escolarización, ya que este tipo de estudios se ha desarrollado solo incidentalmente (Arnold et al., 2005).

En términos estrictamente educativos, la identificación y caracterización de perfiles de maestros rurales efectivos, es de gran importancia. Dado que se ha demostrado el vínculo entre la calidad de los maestros y el logro del estudiante (Arnold et al., 2005; Pajares, 2003), la necesidad de una guía basada en la evidencia, relativa a la competencia docente, la preparación y el desarrollo profesional, resulta vital para los tomadores de decisiones respecto de la educación rural. 
Por último, la elección de la escuela en las zonas rurales es un tema que ha sido pasado por alto en las investigaciones. Esta brecha en la base de conocimiento de la educación rural, puede deberse a la percepción de que el aislamiento geográfico se opone a la elección, sin embargo, a diferencia de lo que ocurre en la educación urbana, no existe evidencia al respecto (Arnold et al., 2005).

En definitiva, esta nueva información, que complementaría los aportes del presente estudio, permitiría mejorar la educación rural actual, especialmente en los sectores más pobres, respondiendo al desafío que conlleva el problema de la desigualdad social. Contar con esta evidencia, permitiría desarrollar más y mejores líneas de acción, que compensen las desventajas de los estudiantes más pobres, y mejoren la calidad de los procesos de aprendizaje a los que acceden los niños y niñas que habitan las zonas rurales del país.

\section{Referencias}

Áberg-Brentsson, L. (2009). The smaller the better? A review of research on small schools in Sweden. International Journal of Educational Research, 48(29), 100-108.

Álvarez, L., Álvarez-García, D., González-Castro, P., Núñez, J., \& González-Pienda, J. (2006). Evaluación de los comportamientos violentos en los centros educativos. Psicothema, 18(4), 686-695.

Anderson, J. (2005). Accountability in Education. Bruselas: UNESCO, IIEP, IAE.

Anderson, J., Chui, M., \& Yore, L. (2010). International perspectives on successes and challengers: Research and policy directions. International Journal of Science and Mathematics Education, 8(3), 593-609.

Araya, F., Dell, R., Donoso, P., Marianov, V., Martínez, F., \& Weintraub, A. (2012). Optimizing location and size of rural school in Chile. International Transactions in Operational Research, 19(5), 695. 710.

Arnold, M., Newman, J., Gaddy, B., \& Dean, C. (2005). A look at the condition of rural education research: setting a direction for future research. Journal of Research in Rural Education, 20(6), 1-23.
Arón, A., Milicic, N., \& Armijo, I. (2012). Clima Social Escolar: una escala de evaluación -Escala de Clima Social Escolar, ECLIS-. Universitas Psychologica, 11(3), 803-813.

Arshad, M., Ahmad, R., Tahira, R., \& Ahmad, F. (2011). Impact of Teacher Quality on the Academic Achievement. European Journal of Social Sciences, 19(1), 97-105.

Atchoarena, D. \& Gasperini, L. (2004). Educación para el desarrollo rural: hacia nuevas respuestas de política. Paris, Francia: FAO - UNESCO.

Ávila, M. (2010). Estudio caracterización de las escuelas rurales multigrado de la región del Maule basado en el modelo de calidad de la gestión escolar. Revista Digital eRural, Educación, cultura y desarrollo rural, 7(13 y 14), 1-127.

Barley, Z., \& Beesley, A. (2007). Rural school success: what can we learn? Journal of Research in Rural Education, 22(1), 1-14.

Boix, R. (2003). Escuela rural y territorio: entre la desruralización y la cultura local. Revista Digital eRural, Educación, Cultura y Desarrollo Rural. Recuperado de http:// www.red-ler.org/escuela-rural-territorio. pdf

Brady, R. (2003). Can Failing Schools be fixed? Washington, DC: Thomas B. Fordham Foundation.

Breiman, L., Freidman, J., Olshen, R., \& Stone, C. (1984). Classification and Regression Trees. Belmont, CA: Wadsworth International Group.

Carmichael, C., \& Taylor, J. (2005). Analysis of student beliefs in a tertiary preparatory mathematics course. International Journal of Mathematical Education in Science and Technology, 36(7), 713-719.

Carnoy, M., \& Loeb, S. (2002). Does external accountability affect student outcomes? A cross-state analysis. Educational Evaluation and Policy Analysis, 24(4), 305-331.

Coll, R., Dahsah, C., \& Faikhamta, C. (2010). The influence of educational context on science learning: a cross-national analysis of PISA. Research in Science EO Technological Education, 28(1), 3-24.

Contreras, P., \& Corbalán, F. (2010). iQué podemos esperar de la Ley de Subvención Escolar Preferencial? Revista de Docencia, (41), 5-16.

Cordero, J., Crespo, E., \& Santín, D. (2010). Factors affecting educational attainment: evidence form 
Spahish PISA 2006 Results. Regional and Sectorial Economics Studies, (10), 3. Recuperado de http:// www.usc.es/ economet/reviews/eers1034.pdf

Corvalán, J. (2004). Síntesis y análisis global de resultados por países. En Educación para la población rural en Brasil, Chile, Colombia, Honduras, México, Paraguay y Perú. Proyecto FAO-UNESCO-DGCS/ ITALIA-CIDE-REDUC. Roma: FAO-UNESCO.

Chiang, H. (2009). How accountability pressure on failing schools affects student achievement. Journal of Public Economics, 93(9-10), 1045-1057.

Cuthrell, K., Stapleton, J., \& Ledford, C. (2010). Examining the culture of poverty: Promising practices. Preventing School Failure, 54(2), 104-110.

De Jorge, J., \& Santín, D. (2010). Los determinantes de la eficiencia educativa den la Unión Europea. Hacienda Pública Española. Revista de Economía Pública, 193(2), 131-156.

Delich, A., \& Iaies, G. (2009). Los sistemas educativos y cohesión social: la reconstrucción de lo común en los estados nacionales del siglo XXI. En C. Cox \& S. Schwartzman (Eds.), Politicas educativas y la cohesión social en América Latina (pp.175-230). Santigo: Uqbar Editores.

Demir, I., \& Depren, Ö. (2010). Assessing Turkey's secondary schools performance by different region in 2006. Procedia - Social and Behavioral Sciences, 2(2), 2305-2309.

Donoso, S., \& Arias, O. (2012). Distribución desigual de las oportunidades educativas en el territorio y migración de la matrícula escolar: el caso de la región de Los Lagos (Chile). Estudios Pedagógicos, 38(2), 35-54.

Duston, J. (2002). El capital social campesino en la gestión del desarrollo rural. Santiago de Chile: Comisión Económica para América Latina y el Caribe, CEPAL.

Elacqua, G., \& Martínez, M. (2011). Searching for schools in a low quality market: evidence from Chile. Working Paper №1. Santiago de Chile: Instituto de Políticas Públicas, Universidad Diego Portales.

Elacqua, G., Santos, H., Salazar, F., \& Brunner, J. (2011). ¿Estamos preparados para cerrar las malas escuelas en Chile? Impacto sobre equidad en el acceso a educación de calidad. Proyecto FONIDE F511083.
Elacqua, G., Martínez, M., Santos, H., \& Urbina, D. (2013). Escuelas bajo amenaza: efectos de corto plazo de las presiones de accountability de la ley SEP en las políticas y prácticas docentes. Documento de trabajo №16, ISSN 0719-4056. Instituto de Políticas Públicas Facultad de Economía y Empresa, Universidad Diego Portales.

Fantuzzi, J. (2008). Competencia y Resultados Educacionales en los Colegios Rurales de Chile. (Tesis de Maestría en Economía). Pontificia Universidad Católica de Chile, Santiago de Chile.

Feu, J. (2003). Escuela rural: apuntes para un debate. Cuadernos de Pedagogía. (327), 90-94.

Figlio, D., \& Rouse, C. (2006). Do accountability and voucher threats improve low- performing schools? Journal of Public Economics, 90, 239-255.

Gallego, F., Rodríguez, C., \& Sauma, E. (2007). Provisión de Educación en Zonas Rurales de Chile: Incentivos, Costos y Calidad. Santiago de Chile: Pontificia Universidad Católica de Chile.

Gallego, F., Rodríguez, C., \& Sauma, E. (2010). The Political Economy of School Size: Evidence from Chilean Rural Areas. Santiago de Chile: Pontificia Universidad Católica de Chile.

García-Hierro, M., \& Cubo, S. (2009). Convivencia escolar en Secundaria: aplicación de un modelo de mejora del clima social. Revista Electrónica Interuniversitaria de Formación del Profesorado, 12(1), 51-62.

Gershberg, A., González, P. \& Meade, B. (2012). Understanding and Improving Accountability in Education: A Conceptual Framework and Guideposts from Three Decentralization Reform Experiences in Latin America. World Development, 40(5), 1024-1041.

Gershberg, A., Meade, B., \& Andersson, S. (2009). Providing better education services to the poor: Accountability and context in the cast of Guatemalan decentralization. International Journal of Educational Development, 29(3), 187-200.

Hanushek, E., \& Raymond, M. (2005). Does school accountability lead to improved student performance? Journal of Policy Analysis and Management, 24(2), 297-327.

Hargreaves, L. (2009). Respect and responsibility: Review of research on small rural schools in England. 
International Journal of Educational Research, 48(2), 117-128.

Hargreaves, L., Kvalsund, R., \& Galton, M. (2009). Reviews of research on rural schools and their communities in British and Nordic countries: Analytical perspectives and cultural meaning. International Journal of Educational Research, 48(2), 80-88.

Hopson, L., \& Lee, E. (2011). Mitigating the effect of family poverty on academic and behavioral outcomes: The role of school climate in middle and high school. Children and Youth Service Review, 33(11), 2221-2229.

Hotelling, H. (1933). Analysis of a complex of statistical variables into principal components. Journal of Educational Psychology, 24(6), 417-441.

Jacob, B. (2005). Accountability, incentives, and behavior: the impact of high-stakes testing in the Chicago public schools. Journal of Public Economics, 89(5-6), 761-796.

Kalaoja, E., \& Pietarinen, J. (2009). Small rural primary schools in Finland: A pedagogically valuable part of the school network. International Journal of Educational Research, 48(2), 109-116.

Kalender, I., \& Berberoglu, G., (2009). An assessment of factors related to science achievement of Turkish students. International Journal of Science Education, 31(10), 1379-1394.

Kearns, R., Lewis, N., McCreanor, T., \& Witten, K. (2009). 'The status quo is not an option': community impacts of school closure in South Taranaki. New Zealand. Journal of Rural Studies, 25(1), 131 140.

Kim, J., \& Sunderman, G. (2004). Does NCLB provide good choices for students in low performing schools? Cambridge, MA: The Civil Rights Project at Harvard University.

Kovács, K. (2012). Rescuing a small village school in the context of rural change in Hungary. Journal of Rural Studies, 28(1), 108-117.

Meneses, J., \& Mominó, J. (2008). La generación de capital social en el sistema educativo no universitario: un análisis del desarrollo comunitario de las escuelas de Cataluña. Papers. (87), 47-75.
Ministerio de Educación de Chile (2008). Ley №20.248 de la Subvención Escolar Preferencial. Santiago de Chile: Autor.

Ministerio de Educación de Chile. (2010). Directorio de Establecimientos Educacionales. Recuperado de http://www.fonide.cl/DedPublico/archivos_ de_datos.

Ministerio de Educación de Chile (2011). Datos SIMCE, Santiago, Chile: Autor. Recuperado de www. simce.cl

Mizala, A., \& Romaguera, P. (2000). School performance and choice: The Chilean experience. The Journal of Human Resources, 35(2), 392-417.

Moreno, D., Estévez, E., Murgui, S., \& Musitu, G. (2009). Relación entre el clima familiar y el clima escolar: el rol de la empatía, la actitud hacia la autoridad y conducta violenta en la adolescencia. International Journal of Psychological Therapy, 9(1), 123-136.

Núñez, C., Solís, C., \& Soto, R. (2014). ¿Qué sucede en las comunidades cuando se cierra la escuela rural? Un análisis psicosocial de la política de cierre de las escuelas rurales en Chile. Universitas Psychologica, 13(2), 615-625.

OECD (Ed.). (2011). Against the odds: Disadvantaged students who succeed in school. Paris: OECD Publishing. Retrieved from http://dx.doi. org/10.1787/9789264090873-en

Oreiro, C., \& Valenzuela, J. (2011). Factores Determinantes del Desempeño Educativo de Uruguay 2003-2006. Serie de Documentos de Trabajo №340, Universidad de Chile, Departamento de Economía.

Pajares, F. (2003). Self-efficacy beliefs, motivation and achievement in writing. Reading and Writing Quarterly, 19(2), 139-158.

Perticara, M., Román, M., \& Selman, J. (2013). Rendimiento escolar y uso de los recursos de la ley SEP. Documento de Investigación $N^{\circ} 297$. Facultad de Economía y Negocios. Universidad Alberto Hurtado.

Raczynski, D, Wieinstein, J., \& Pascual, J. (2013). Subvención Escolar Preferencial (SEP) en Chile: Un Intento por Equilibrar la Macro y Micro Política Escolar. Revista Iberoamericana sobre Calidad, Eficacia y Cambio en Educación, 11(2), 164-193. 
R-Development Core Team (2011). R: A language and environment for statistical computing. Vienna, Austria: the R Foundation for Statistical Computing.

Reback, R. (2008). Teaching to the rating: school accountability and the distribution of student achievement. Journal of Public Economics, 92(56), 1394-1415.

Riella, A., \& Vitelli, R. (2005). Escuelas rurales y desarrollo territorial: una reflexión para el caso Uruguayo. Revista Pampa. (1), 1-20.

Sherwood, T. (2000). Where has all the "rural" gone? Rural education research and current federal reform. Journal of Research in Rural Education, 16(3), 159-167.

Schunk, D. (2003). Self-efficacy for reading and writing: Influence of modeling, goal setting and self-evaluation. Reading and Writing Quarterly: Overcoming Learning Difficulties, 19(2), 159-172.

SIMCE. (2011). Resultados Nacionales SIMCE 2010. Ministerio de Educación de Chile. Recuperado de http://www.simce.cl/

Smarick, A. (2010). The turnaround fallacy. Education Next, 10(1), 20-26.

Smit, R., \& Humpert, W. (2012). Differentiated instruction in small schools. Teaching and Teacher Education, 28(8), 1152-1162.

Spreng, C. (2005). Policy Options for Interventions in Failing Schools. U.S.A: Rand Corporation.

Springer, M. (2008). The influence of an NCLB accountability plan on the distribution of student test score gains. Economics of Education Review, 27(5), 556-563.
Valenzuela, J., \& Allende, C. (2014). Trayectorias de Mejoramiento en el Sistema Escolar Chileno: Las Escuelas de Educación Básica 2002-2010. Apuntes sobre Mejoramiento Escolar №1. Centro de Investigación Avanzada en Educación, CIAE. Universidad de Chile.

van Dinther, M., Dochy, F., \& Segers, M. (2011). Factors affecting students' self-efficacy in higher education. Educational Research Review, 6(2), 95-108.

Vera, D., Osses, S., \& Shiefelbein, E. (2012). Las creencias de los profesores rurales: una tarea pendiente para la investigación educativa. Estudios Pedagógicos, 38(1), 311-324.

Vera, D. (2013). iVale la pena mantener las escuelas rurales abiertas? (Tesis doctoral inédita). Universidad de La Frontera, Temuco, Chile.

Vera, D., Salvo, S., \& Zunino, H. (2013). En torno al cierre de Escuelas Rurales en Chile. Antecedentes para la implementación de una política de Estado. Investigaciones en Educación. 13(1), 123-143. Recuperado de http://dungun.ufro.cl/ mageduc/docs/ rie_2013vol1.pdf

Villalta, M., \& Saavedra, E. (2012). Cultura escolar, prácticas de enseñanza y resiliencia en alumnos y profesores de contextos sociales vulnerables. Universitas Psychologica, 11(1), 67-78.

West, M., \& Peterson, P. (2006). The efficacy of choice threats within school accountability systems: results from legislatively induced experiments. Economic Journal, 116(510), 46-62.

Willms, J. (2010). School composition and contextual effects on student outcomes. Teachers College Record, $112(4), 1008-1037$. 